\title{
On the Efficacy of Isolating Shoulder and Elbow Movements with a Soft, Portable, and Wearable Robotic Device
}

\author{
Zahra Kadivar, Christopher E. Beck, Roger N. Rovekamp, Marcia K. O'Malley, and Charles A. Joyce
}

\begin{abstract}
Treatment intensity has a profound effect on motor recovery following neurological injury. The use of robotics has potential to automate these labor-intensive therapy procedures that are typically performed by physical therapists. Further, the use of wearable robotics offers an aspect of portability that may allow for rehabilitation outside the clinic. The authors have developed a soft, portable, lightweight upper extremity wearable robotic device to provide motor rehabilitation of patients with affected upper limbs due to traumatic brain injury (TBI). A key feature of the device demonstrated in this paper is the isolation of shoulder and elbow movements necessary for effective rehabilitation interventions. Herein is presented a feasibility study with one subject and demonstration of the device's ability to provide safe, comfortable, and controlled upper extremity movements. Moreover, it is shown that by decoupling shoulder and elbow motions, desired isolated joint actuation can be achieved.
\end{abstract}

\section{INTRODUCTION}

$\mathrm{C}$ URRENT rehabilitation research indicates that taskspecific [1] and intensive [2] practice could significantly improve motor recovery and induce neuroplasticity after brain injury. The greater effectiveness of intensive task-specific practice relative to standard therapy techniques suggests that repetitive motor practice is a crucial rehabilitation component, and provides a key opportunity for the introduction of robotics in rehabilitation. Achieving voluntary isolated movements after neurological injuries such as TBI and stroke is a common therapy goal. Synergistic movement patterns that occur after neurological injuries have the potential to interfere with activities of daily living (ADLs) and limit functional independence of the individual. A common rehabilitation approach to address unwanted synergies includes manual fixation of undesired movements and facilitation of desired movements.

The trend of applying robotics to address these rehabilitation challenges continues to grow. Existing robotic devices [3-5] that provide the capability to assist the affected proximal upper extremities for rehabilitative purposes, however, are ground-based. Soft, portable, wearable robotic devices offer additional potential advantages, including allowing more task-oriented therapy (i.e. performing functional tasks as opposed to merely prescribed motions that are somewhat decoupled from practical ADLs), and bringing therapy to new venues including the home.

This work was funded by the DARPA Warrior Web program (BAA-1343) and NASA Grant NNX14AK51G.

Z. Kadivar and M. K. O'Malley are with Rice University, Houston, USA (zkadivar@gmail.com, omalleym@ rice.edu).
The authors developed a soft upper extremity wearable robotic device at the NASA JSC Wearable Robotics Laboratory for the purpose of providing effective upper extremity motor rehabilitation related to TBI, a condition that has left approximately 5.3 million Americans with a longterm disability [6]. In this paper, a feasibility study with a single subject is presented to demonstrate successful isolation of upper extremity movements with a soft wearable robotic device. Given the portable nature of the device, performance of these rehabilitative movements can occur in the modified settings and task-specific situations alluded to above, potentially offering a richer approach to the rehabilitation of upper extremity function.

\section{MATERIALS AND METHODS}

\section{A. Device Description}

The novel soft, portable, wearable robotic device (see Fig. 1) actively controls the right shoulder and elbow, both positioning the limb in specific orientations and commanding the limb through desired motions. The device uses a minimal amount of rigid components and custom force-controllable tendon actuators developed at NASA JSC. While the system currently only actuates the right arm, it was designed to easily incorporate a left arm in the future.

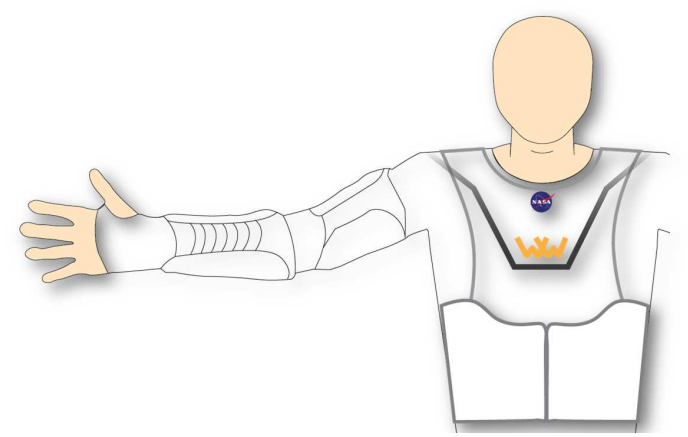

Fig. 1. An artist's depiction of the soft upper extremity wearable robotic device developed at NASA JSC for motor rehabilitation related to TBI.

Careful design consideration was given to ease donning and doffing of the device, both to minimize valuable patient and physical therapist time and to maximize patient comfort, especially in the presence of muscle tone. Moreover, comfort of the patient throughout the session was a priority in the

C. E. Beck, R. N. Rovekamp, and C. A. Joyce are with the NASA JSC Wearable Robotics Laboratory, Houston, USA (christopher.e.beck, roger.n.rovekamp, charles.a.joyce@ nasa.gov). 
design, resulting in a physical human-robot interface that effectively distributes loads around the torso, minimizing pressure points and interface migration.

The device offers multiple control modes. In active assist mode, shoulder abduction and flexion, and elbow flexion, may be commanded either simultaneously via coordinated control or individually while holding position/orientation of the other joints. In passive assist mode, the user may freely move his or her limb while the system provides minimal torque to the shoulder and elbow degrees of freedom.

\section{B. Subject Description}

Evaluation of the device was conducted with a single subject, male, age 29, with moderate TBI, 1.5 years postinjury. Due to right hemiparesis the subject was unable to achieve full ranges of shoulder abduction and flexion against gravity. However, the subject was able to achieve full elbow flexion against gravity and minimal resistance. Compensatory movements were present during active shoulder and elbow movements leading to synergistic patterns (e.g. shoulder abduction and internal rotation during elbow flexion). Increased tone was present in the muscles controlling elbow flexion, finger flexion, and shoulder internal rotation of the affected limb.

\section{Exercise Description}

Due to the subject's inability to isolate movements, a commanded isolated active elbow flexion/extension trajectory was chosen while holding a commanded position/orientation of the shoulder. This allowed for the evaluation of the utility of the device in supporting movement isolation. In the first trial, the subject was asked to follow a commanded elbow joint angle trajectory between $30^{\circ}$ and $90^{\circ}$ at a constant velocity of $\sim 13 \%$. The subject was instructed to only move the elbow joint. During this trial, minimum shoulder and elbow joint torque was applied via the actuators such that the subject was responsible for performing the motion with no assistance from the device. In the second trial, the subject was asked to perform the same movement while the device maintained the shoulder in an abducted position. The subject was provided with real-time visual feedback of the desired and actual elbow angles during all trials.

\section{RESULTS}

The testing session lasted approximately one hour, without any adverse events or reports of discomfort from the subject. The actuated range of motion (intentionally constrained to avoid exceeding the patient's passive range of motion) was demonstrated for shoulder abduction, flexion, and elbow flexion. In active assist mode, the device properly positioned the limb at the commanded orientations.

The subject used compensatory shoulder abduction to achieve desired elbow range of motion (as seen in Figure 2). With robot-assisted fixation of the shoulder, a similar range of elbow movement was achieved. However, compensation from shoulder abduction was greatly reduced. Fixation of the shoulder not only allowed for the ability to move the elbow joint in isolation, but it also improved smoothness of the movement, as joint velocity error decreased from the first trial, during which the shoulder was free.

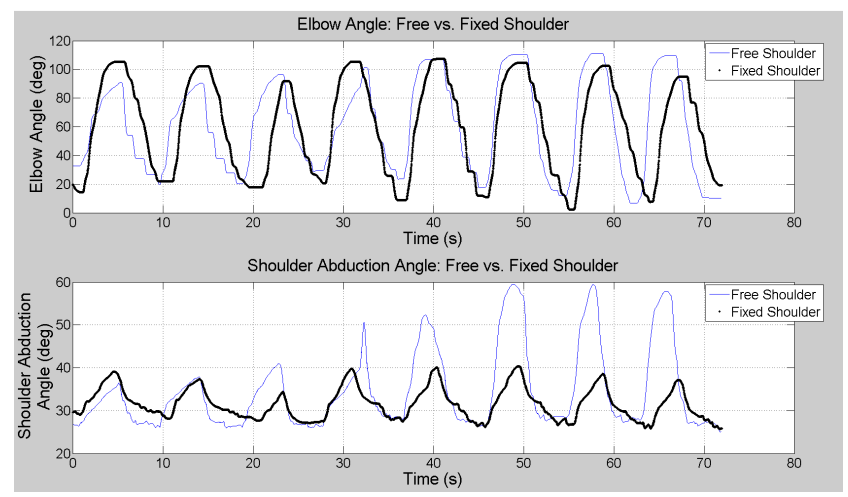

Fig. 2. Compensatory shoulder abduction is evident during elbow movement when shoulder is free (Trial 1; blue line). Shoulder fixation (Trial 2; black line) allows isolation of elbow and shoulder movements.

\section{CONCLUSION}

The results demonstrate the feasibility of isolating upper extremity movements with a soft, portable, wearable robotic device. The current study indicates the possibility of using the developed wearable robotic device for improving motor control and coordination of the upper extremity after traumatic brain injury. Although rehabilitation was not directly addressed, unique control features and the portability, wearability, and comfort of the device highlight its potential for upper limb rehabilitation. In light of this, such a device could pave the way to more task-based forms of therapy and provide a medium for increased dosage. Additional potential applications of the device are already under investigation, including assist-as-needed control of the upper extremity and assistance with ADLs. The desire to provide both beneficial rehabilitation and practical ADL assistance with the same hardware is well aligned with the capabilities of a soft, portable, wearable robotic device.

\section{REFERENCES}

[1] I. J. Hubbard, M. W. Parsons, C. Neilson, and L. M. Carey, "Taskspecific training: evidence for and translation to clinical practice," Occup. Ther. Int., vol. 16, no. 3-4, pp. 175-189, Sep. 2009.

[2] A. Sunderland and A. Tuke, "Neuroplasticity, learning and recovery after stroke: a critical evaluation of constraint-induced therapy," Neuropsychol. Rehabil., vol. 15, no. 2, pp. 81-96, 2005.

[3] T. Nef, M. Mihelj, G. Kiefer, C. Perndl, R. Müller, and R. Riener, "ARMin - Exoskeleton for Arm Therapy in Stroke Patients," in IEEE Int. Conf. Rehab. Rob., Noordwijk, The Netherlands, 2007, pp. 68-74.

[4] H. I. Krebs, N. Hogan, M. L. Aisen, and B. T. Volpe, "Robot-Aided Neurorehabilitation," IEEE Trans. Rehabil. Eng., vol. 6, no. 1, pp. 7587, Mar. 1998.

[5] C. G. Burgar, P. S. Lum, P. C. Shor, and H. F. M. Van der Loos, "Development of robots for rehabilitation therapy: the Palo Alto VA/Stanford experience," J. Rehabil. Res. Dev., vol. 37, no. 6, pp. 663673, Nov. 2000.

[6] Injury Prevention \& Control: Traumatic Brain Injury \& Concussion. (n.d.). Center for Disease Control and Prevention. [Online]. http://www.cdc.gov/traumaticbraininjury/severe.html. Accessed Apr. $1,2016$. 